\title{
Curvature Effects on Surface Electron States in Ballistic Nanostructures
}

\author{
Hisao Taira and Hiroyuki Shima \\ Department of Applied Physics, Graduate School of Engineering, Hokkaido \\ University, Sapporo 060-8628 Japan
}

\begin{abstract}
The curvature effect on the electronic states of a deformed cylindrical conducting surface of variable diameter is theoretically investigated. The quantum confinement of electrons normal to the curved surface results in an effective potential energy that affects the electronic structures of the system at low energies. This suggests the possibility that ballistic transport of electrons in low-dimensional nanostructures can be controlled by inducing a local geometric deformation.
\end{abstract}

Key words: surface electron states, curved surface, geometrical effects PACS: 73.20.At, 73.21.Hb, 73.22.Dj

\section{Introduction}

Recent advances in the manipulation of nanostructures have enabled the fabrication of reduced-dimensional quantum systems with novel geometry [1-11]. The understanding of their basic properties and the accurate modeling of their electronic structures are of vital importance for the manufacture of nanodevices and their applications. From the theoretical viewpoints, the peculiar features exhibited by quantum systems confined to low-dimensional curved geometry are of interest. These peculiarities arise from an interplay between geometry and quantum physics; in fact, when the electron is strongly confined to a smoothly curved surface, it experiences an effective potential energy whose magnitude depends on the local curvatures along the surface [12-24]. Due to this curvature effect, the electrons can not move around freely on the surface even in the absence of impurities or other interacting entities. This implies that

Email address: taira@eng.hokudai.ac.jp, shima@eng.hokudai.ac.jp (Hisao Taira and Hiroyuki Shima).

Preprint submitted to Elsevier $\quad 7$ October 2018 
the quantum transport of low-dimensional nanostructures can be controlled by altering the local geometric curvature.

In the present work, we theoretically investigate the curvature effect on the quantum properties of electrons confined to a cylindrical surface. The local deformation of the cylindrical surface significantly affected the spatial profile of the electron eigenstates in the lowest-energy region. This indicates the occurrence of a curvature-induced alteration in the ballistic electron transport in nanoscale cylindrical surfaces.

\section{The confining potential approach}

In quantum mechanics, the motion of quantum particles constrained to a twodimensional curved surface is described by one of the two formalisms given below. One is the intrinsic quantization approach, in which the motion is constrained to the surface a priori; namely, a classical Hamiltonian is firstly constructed from coordinates and momentum intrinsic to the surface, following which the system is quantized canonically. The other is the confining potential approach, in which the particle is assumed to be confined by a strong force that acts normal to the curved surface. In this approach, the quantization of the motion perpendicular to the curved surface results in an effective potential that depends on the local surface curvature. Among the two formalisms, we employ the latter one since it offers a physically more realistic model of quantum confinement to curved surfaces. (In fact, in any real physical system, constrained motion is the result of a strong confining force.) It is mentioned that the confining potential approach was initially suggested by da Costa [13] ; it has been successfully applied to quantum mechanical problems involving novel geometries [25-30].

Let $\left(x^{1}, x^{2}, x^{3}\right)$ be a three-dimensional curvilinear coordinate. This allows the parameterization of a curved surface of interest by $\boldsymbol{r}=\boldsymbol{r}\left(x^{1}, x^{2}\right)$. Then, a point $\boldsymbol{p}$ in this space can be determined through the relation

$$
\boldsymbol{p}=\boldsymbol{r}\left(x^{1}, x^{2}\right)+x^{3} \boldsymbol{n}\left(x^{1}, x^{2}\right),
$$

where $\boldsymbol{n}\left(x^{1}, x^{2}\right)$ is the vector normal to the curved surface. We now introduce a confining potential $V\left(x^{1}, x^{2}, x^{3}\right)$. After a proper limiting procedure [13], the potential becomes $V=0$ if $x^{3}=0$ and $V=\infty$ otherwise. This allows us to separate the $x^{3}$ dependence in the Hamiltonian of the confined system and eventually provides the Schrödinger equation for curved surfaces:

$$
-\frac{\hbar^{2}}{2 m^{*}}\left[\frac{1}{\sqrt{g}} \frac{\partial}{\partial x^{i}}\left(\sqrt{g} g^{i j} \frac{\partial}{\partial x^{j}}\right)-\left(H^{2}-D\right)\right] \sigma=E \sigma,
$$




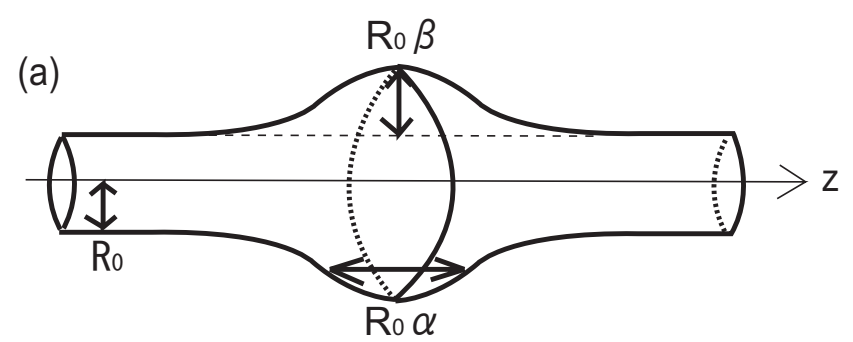

(b)

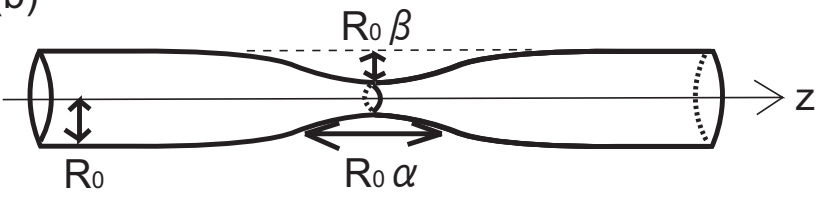

Fig. 1. Schematic illustration of a deformed cylindrical surface. The parameter $\alpha$ determines the spatial extent of the deformed part along the $z$-axis, and $\beta$ determines the extent of a bulge $(\beta>0)$ or constriction $(\beta<0)$.

where,

$$
g_{i j}=\frac{\partial \boldsymbol{r}}{\partial x^{i}} \cdot \frac{\partial \boldsymbol{r}}{\partial x^{j}}, \quad g=\operatorname{det}\left[g_{i j}\right] \quad \text { and } \quad g^{i j}=\left[g_{i j}\right]^{-1} .
$$

In Eq. (2), $\sigma\left(x^{1}, x^{2}\right)$ is the wave function of the confined particles, and $H\left(x^{1}, x^{2}\right)$ and $D\left(x^{1}, x^{2}\right)$ are the mean and Gaussian curvatures of the surface, respectively [31]. The occurrence of the non-trivial potential term, $-\left(H^{2}-D\right)$ in Eq. (2), is a direct consequence of the quantization of the motion normal to the surface. It is emphasized that this potential term depends only on the local surface geometry and not on the mass or charge of the particle.

\section{Model and methods}

\subsection{Schrödinger equations for deformed cylindrical surfaces}

Figure 1 gives a schematic illustration of a cylindrical surface subject to local deformation. ${ }^{1}$ This curved surface is parameterized by

$$
\boldsymbol{r}=\boldsymbol{r}(z, \phi)=[R(z) \cos \phi, R(z) \sin \phi, z]
$$

where the radius of the cylinder is assumed to vary with $z$ as

$$
R(z)=R_{0}\left[1+\beta \exp \left(\frac{-2 z^{2}}{\alpha^{2} R_{0}^{2}}\right)\right] .
$$

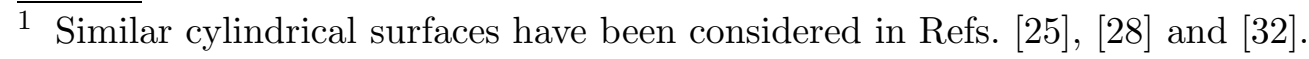


Hence, the geometry of the surface in question is determined by the two parameters: the parameter $\alpha$ determines the spatial extent of the deformed part along the $z$-axis, and $\beta$ determines the extent of a bulge $(\beta>0)$ or constriction $(\beta<0)$.

Our current objective is to deduce the electron eigenstates of the curved surfaces introduced above. It is noteworthy that, due to the rotational symmetry of the surfaces, the Schrödinger equation (2) can be further simplified by means of the variable-separation method. This is seen by substituting

$$
\sigma(z, \phi)=\frac{\eta(z)}{\sqrt{R(z) f(z)}} \frac{e^{i m \phi}}{\sqrt{2 \pi}}
$$

into Eq. (2), with the definition

$$
f(z)=\frac{1}{\sqrt{1+(d R / d z)^{2}}} .
$$

Then, we obtain the reduced Schrödinger equation for $\eta(z)$ as

$$
-\frac{\hbar^{2}}{2 m^{*}}\left[f(z)^{2} \frac{d^{2} \eta}{d z^{2}}+v(z) \eta(z)\right]=\epsilon \eta(z)
$$

where

$$
v(z)=f(z)^{2}\left[\frac{d \Gamma}{d z}-\Gamma(z)^{2}\right]+H(z)^{2}-D(z)-\frac{m^{2}}{R(z)^{2}},
$$

and

$$
\begin{aligned}
& \Gamma(z)=-\frac{1}{2 R(z) f(z)} \frac{d(R f)}{d z} \\
& H(z)=f(z)^{3} \frac{d^{2} R}{d z^{2}}-\frac{f(z)}{R(z)} \\
& D(z)=-\frac{f(z)^{3}}{R(z)} \frac{d^{2} R}{d z^{2}}
\end{aligned}
$$

Consequently, the problem is reduced to solving the differential equatioin (8) with respect to $\eta(z)$. By observing the $\alpha$ - and $\beta$ - dependences of $\eta(z)$, we can clarify the curvature effect on the electronic structures of deformed cylindrical surfaces. 


\subsection{Tight-binding approximation}

In the actual calculations in Eq. (8), we have employed the tight-binding approximation; i.e., the continuous variable $z$ is discretized into a set of discrete numbers $i(1 \leq i \leq N)$ with an equiseparation $a$. The resulting difference equation is

$$
t_{0}\left(2 f_{i}^{2}-v_{i}\right) \eta_{i}-t_{0} f_{i}^{2}\left(\eta_{i+1}+\eta_{i-1}\right)=\epsilon \eta_{i}
$$

where the constant $t_{0} \equiv \hbar^{2} /\left(2 m^{*} a^{2}\right)$ denotes the hopping energy between neighboring sites in an undeformed region. Nonzero surface curvature at the deformed region manifest itself in spatial modulation of $f_{i}$ and $v_{i}$ given in Eq. (13). In fact, $f_{i} \equiv 0$ and $v_{i} \equiv$ const. if the cylinder in question is flat (i.e., $R \equiv$ const.).

The differential equation (13) applies to real deformed cylindrical nanosurfaces in which the surface density of constituents (atoms or molecules) is homogeneous over the whole surface. In other words, the separation $r_{i j}$ of neighboring constituents needs to assume nearly constant so that the hopping energy remains to be constant. This condition, however, may violate when an external deformation against the original flat cylindrical surface is magnified. In the latter case, deformation-induced change in $r_{i j}$ can be taken into account by rewriting Eq. (13) as

$$
t_{0}\left(2 f_{i}^{2}-v_{i}\right) \eta_{i}-t_{i i+1} f_{i}^{2} \eta_{i+1}-t_{i i-1} f_{i}^{2} \eta_{i-1}=\epsilon \eta_{i}
$$

Here, $t_{i j}$ represents the spatially dependent hopping energy, which decreases exponentially with the separation $r_{i j}$ as

$$
t_{i j}=t_{0} e^{-\left(r_{i j}-a\right)}
$$

where

$$
r_{i j}=\int_{i}^{j} g_{11}(z) d z, \quad g_{11}(z)=\frac{1}{f(z)^{2}}
$$

Obviously, if $r_{i j} \equiv a$ for all $i$ and $j, t_{i j} \equiv t_{0}$ so that Eq. (14) reduces to Eq. (13). In both the conditions mentioned above, the radius $R_{0}$ of an undeformed part of the cylindrical surface is set to $R_{0}=50$ and the length $L$ of the cylinder is $L=1000$ in units of $a$. 

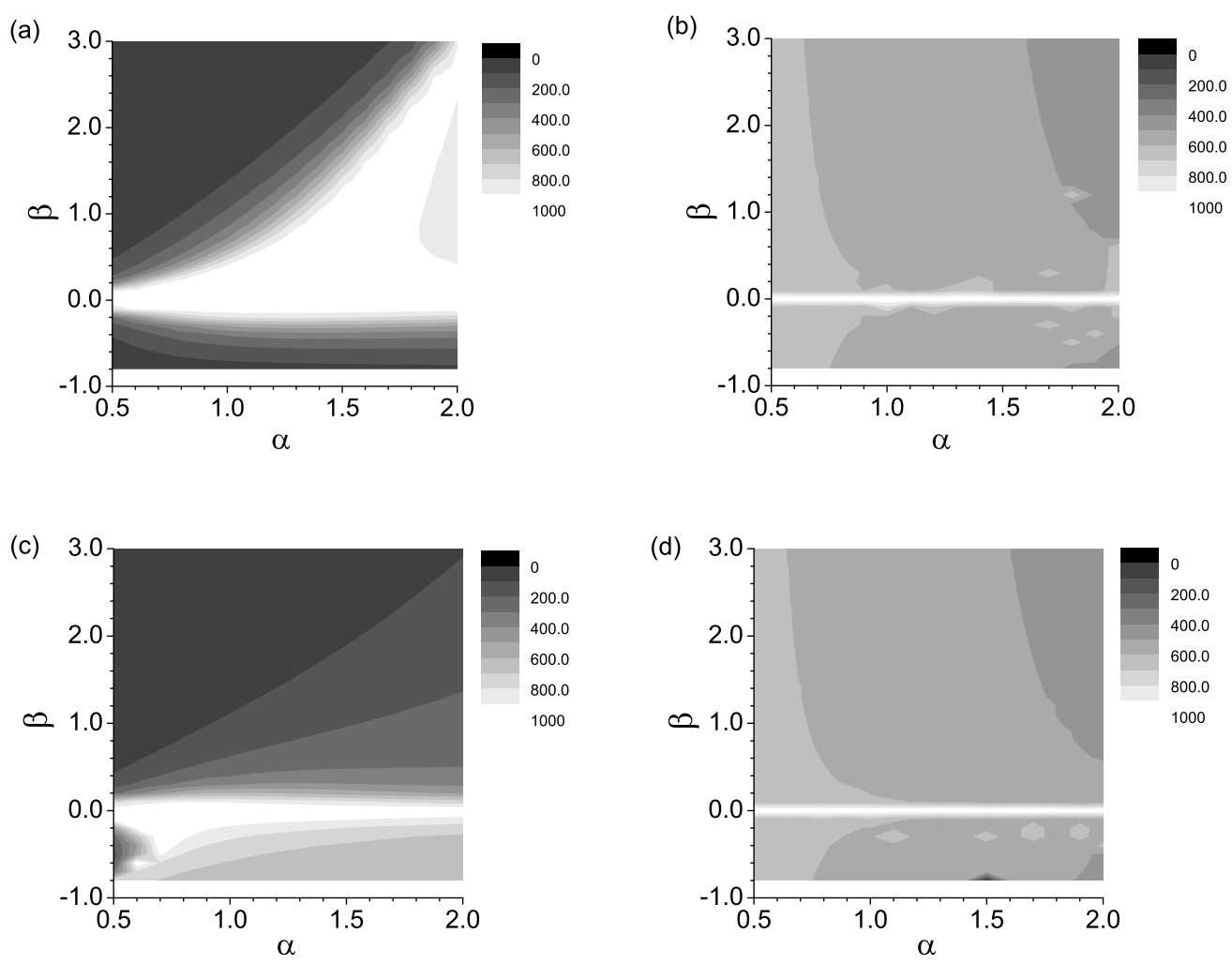

Fig. 2. Contour plots of the localization length $\xi$ of the lowest-energy eigenstate with (a)-(b) $m=0$ and (c)-(d) $m=1$. In the two left panels, the hopping energy $t_{0}$ is assumed to be constant, while in the two right panels, the spatial variation of $t_{i j}$ is taken into account (see Eq. (15)). The eigenstate is strongly localized in the dark region and extends over the entire system in the light region.

\section{Results and discussions}

\subsection{Localization length}

In order to clarify the curvature effect on the spatial profile of $|\eta(z)|^{2}$, we have calculated the localization length $\xi$ of the lowest-energy eigenstate defined by [33]

$$
\xi=\left|\sum_{i=1}^{N} \eta_{i}^{4}\right|^{-1}
$$


This quantity provides a measure of the spatial extent of the wavefunction in question. ${ }^{2}$ Hence, by examining the dependence of $\xi$ on the geometric parameters $\alpha$ and $\beta$, we obtain an understanding of the geometric effect on the electron system on cylindrical surfaces.

Figures 2(a)-(d) show the contour plots of the localization length $\xi$ of the lowest-energy eigenstate with (a)-(b) $m=0$ and (c)-(d) $m=1$. In the two left panels, the hopping energy $t_{0}$ is assumed to be constant (see Eq. (13)), while in the two right panels, the spatial variation of $t_{i j}$ is taken into account (see Eq. (14)). Henceforth, we refer to the two models described by Eqs. (13) and (14) as models A and model B, respectively.

We see from the four panels that the ground-state eigenfunction may be strongly bounded (dark region) or may extend over the entire system (light region) depending on the values of $\alpha$ and $\beta$. We first consider the result of model A; it is evident that in both the cases of $m=0$ (Fig. 2(a)) and $m=1$ (Fig. 2(c)), the $\alpha$ dependence of $\xi$ is completely different in the regions $\beta>0$ (bulging deformation) and $\beta<0$ (constricted deformation). With $m=0$, for instance, $\xi$ for $\beta>0$ tends to increase with $\alpha$ and eventually becomes equal to the system length $L$ at $\alpha \sim 1.5$. This indicates that the ground-state becomes extended $(\xi \sim L)$ when reforming the bulging part to be more stretched in the direction of the $z$-axis. On the other hand, $\xi$ for $\beta<0$ is almost invariant with regard to changes in $\alpha$, and its value is always smaller than $L$. As summarized, (i) the crossover behaviour of $\xi$ from the bounded to extended states is observed only in the case of bulging deformation $(\beta>0)$, and within this range, (ii) the magnitude of $\xi$ for a fixed $\beta$ rapidly increases at $\alpha \sim 1.5$. These two findings suggest the possibility of controlling the ballistic electron transport in cylindrical nanostructures by introducing a subtle geometric deformation.

A similar crossover behaviour of $\xi$ was observed in the case of $m=1$ (Fig. 2(c)). Nevertheless, in contrast to the case of $m=0$, the crossover occurs for negative $\beta$, not for positive. In fact, we have confirmed that when $m \geq 1$, the extended low-energy states occur only at $\beta<0$, which is in contrast to the case of $m=0$, where the extended states occur only at $\beta>0$. This difference as well as the global behavior of $\xi(\alpha, \beta)$ depicted in Figs. 2(a) and 2(c) can be accounted for by considering the contribution from each term in the expression for $v(z)$ (see Eq. (9)); detailed analyses will be presented elsewhere [35,36].

While the localization length $\xi$ in the model $\mathrm{A}$ is responsive to the variations of $\alpha$ and $\beta$, that in model $\mathrm{B}$ exhibits only a slight dependence on the values of $\alpha$ and $\beta$. This is illustrated in Fig. 2(b) for $m=0$ and Fig. 2(d) for $m=1$. In both cases, $\xi$ decreases rather slowly with an increase $\alpha$ and $\beta$, which implies that a geometric deformation has almost no contribution to determining the

$\overline{2}$ Instead of Eq. (17), there is an alternative definition of localization length associated with the Lyapnov exponent; see Ref [34] for example. 

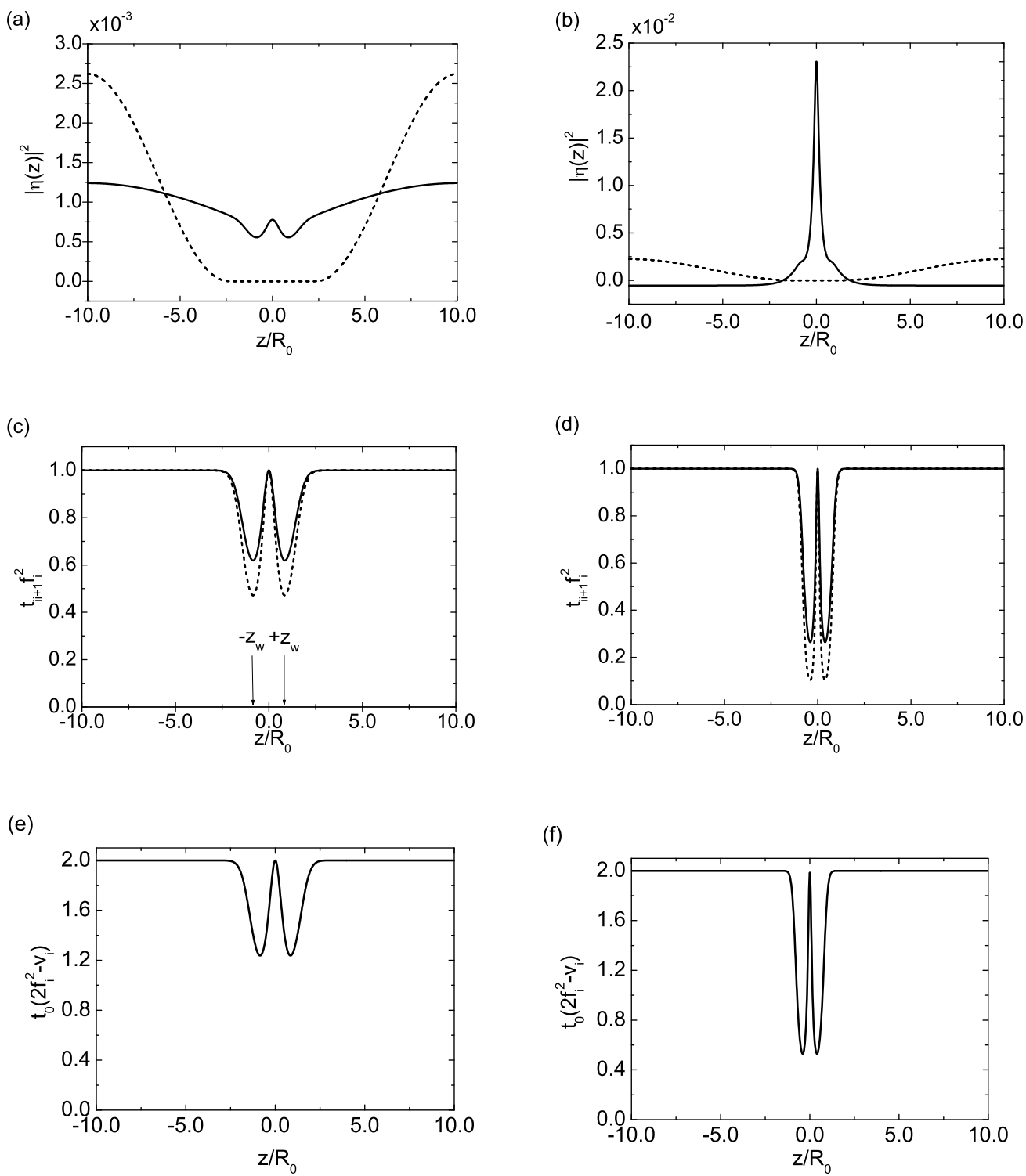

Fig. 3. The upper panels: Spatial profiles of $|\eta(z)|^{2}$ of the ground-state with $m=0$ for model A (the solid line) and for model B (the dotted line). The middle (lower) panels: The $z$-dependence of the off-diagonal (diagonal) element of the Hamiltonian matrix for model $\mathrm{A}$ (solid) and $\mathrm{B}$ (dotted). The parameters are set to be $(\alpha, \beta)=(0.8,1.0)$ in the left panels, and $(\alpha, \beta)=(1.7,1.0)$ in the right panels.

spatial profiles of the eigenstates.

\subsection{Spatial profile of the wavefunction}

To understand the difference in the relevance of geometric deformation to the behavior of $\xi$ between in models $\mathrm{A}$ and $\mathrm{B}$, we extract the spatial profile of 
the square amplitude of the ground-state eigenfunction for $m=0$ by fixing $\alpha$ and $\beta$ to be certain values. Figures 3(a) and 3(b) are plots of the profile of $|\eta(z)|^{2}$ for model A (the solid line) and for model B (the dotted line) with different values of $(\alpha, \beta)$. The parameters are set as $(\alpha, \beta)=(0.8,1.0)$ in the left panels and $(\alpha, \beta)=(1.7,1.0)$ in the right panels. We have also plotted the $z$-dependence of the diagonal and off-diagonal elements of the Hamiltonian matrix for the two models in Figs. $3(\mathrm{c})-(\mathrm{f})$, where $t_{0}$ is taken as units of energy.

We see from Figs. 3(a) and 3(b) that the wavefunction for model A is extended at $\alpha=0.8$, whereas it is spatially bounded around the deformed region $(z \sim 0)$ at $\alpha=1.7$. Indeed, this behavior is completely consistent with the contour plot of $\xi$ depicted in Fig. 2(a). In contrast, the wavefunction corresponding to model $\mathrm{B}$ exhibits almost no variance in its spatial profile for a different value of $\alpha$; it is fairly extended along the $z$-axis with a low (almost negligible) amplitude around the deformed region.

The disappearance of the bound state in model B is qualitatively understood by observing the $z$-dependence of the off-diagonal elements $t_{i j} f_{i}^{2}$, as shown in Figs. 3(c) and 3(d). In both plots, double-well structures whose bottoms are located at $z= \pm z_{w}$ appear. It is noteworthy that the magnitude of the double well of model $\mathrm{B}$ is larger than that of model $\mathrm{A}$. This is because in the model $\mathrm{B}$, the value of the hopping energy $t_{i j}$ at $z= \pm z_{w}$ becomes smaller than $t_{0}$, since the separation $r_{i j}$ becomes larger than $a$ (see Eq. (15)). Then, the quantum hopping across point $\pm z_{w}$ is slightly weak; thus, the wavefunction in model $\mathrm{B}$ tends to have a finite amplitude only within the undeformed region $\left(|z|>z_{w}\right)$ in order to make the energy lower. As a consequence, the geometric deformation in model $\mathrm{B}$ neither engenders spatially bounded eigenstates as done in model A nor contributes significantly to the spatial profile of the lowest-energy eigenstates. This scenario indicates that the low-energy bound states observed in model $\mathrm{A}$ are difficult to realize by inducing an external mechanical deformation to a flat cylindrical conducting surface. We emphasize that the abovementioned findings regarding model B serve as a complementary result to the existing studies of the geometric effect on the electron transport in cylindrical surfaces, where the geometric conditions to realize the bound states were considered $[25,28]$.

\section{Conclusions}

We have investigated the effect of local geometric deformation on the electronic states that are strongly confined to a thin cylindrical surface. The spatial profile of the square amplitude of the lowest-energy eigenstates were numerically evaluated by the tight-binding approach; this was followed by the extraction the localization length of the eigenstate as a function of the geometric param- 
eters $\alpha$ and $\beta$. The results indicate that, under certain conditions, a subtle geometric deformation could induce a drastic change in the ballistic electron transport along nanoscale cylindrical surfaces. We hope that our findings prove to be a fundamental basis for the development of quantum devices based on low-dimensional nanostructures.

\section{Acknowledgements}

We thank T. Nakayama, K. Yakubo and S. Nishino for useful discussions. This work was supported in part by a Grant-in-Aid for Scientific Research from the Japan Ministry of Education, Science, Sports and Culture. One of the authors (HS) is thankful for the financial support from the Murata Science Foundation. HS also acknowledges for the grant from the Mazda Foundation. Numerical calculations were performed in part on the Supercomputer Center, ISSP, University of Tokyo.

\section{References}

[1] W. Tian and S. Datta, Phys. Rev. B 495097 (1994).

[2] A. Lorke, R. J. Luyken, A. O. Govorov, J. P. Kotthaus, J. M. Garcia, and P. M. Petroff, Phys. Rev. Lett. 842223 (2000).

[3] T. Heinzel, K. Ensslin, W. Wegscheider, A. Fuhrer, S. Lüscher, and M. Bichler, Nature (London) 413822 (2001).

[4] A. Latgé, C. G. Rocha, L. A. L. Wanderley, M. Pacheco, P. Orellana, and Z. Barticevic, Phys. Rev. B 67155413 (2003).

[5] S. Latil, S. Roche, and A. Rubio, Phys. Rev. B 67165420 (2003).

[6] A. Goker and P. Norlander, J. Phys.: Condens. Matter 168233 (2004).

[7] D. Gridin, A. T. I. Adamou, and R. V. Craster, Phys. Rev. B 69155317 (2004).

[8] S. X. Qu and M. R. Geller, Phys. Rev. B 70085414 (2004).

[9] Y. Y. Chou, G.-Y. Guo, L. Liu, C. S. Jayanthi, and S. Y. Wu, J. Appl. Phys. 962249 (2004).

[10] M. Sano, A. Kamino, J. Okamura, and S. Shinkai, Science 2931299 (2004).

[11] Y. Pershin and C. Piermarocchi, Phys. Rev. B 72195340 (2005).

[12] H. Jensen and H. Koppe, Ann. Phys. (N.Y.) 63586 (1971).

[13] R. C. T. da Costa, Phys. Rev. A 231982 (1981). 
[14] R. C. T. da Costa, Phys. Rev. A 252893 (1982).

[15] J. Goldstone and R. L. Jaffe, Phys. Rev. B 4514100 (1991).

[16] M. Burgess and B. Jensen, Phys. Rev. A 481861 (1993).

[17] I. J. Clark and A. J. Bracken, J. Phys. A 294527 (1996).

[18] M. Encinosa and B. Etemadi, Phys. Rev. A 5877 (1998).

[19] M. V. Entin and L. I. Magarill Phys. Rev. B 64085330 (2001).

[20] M. V. Entin and L. I. Magarill Phys. Rev. B 66205308 (2002).

[21] M. Encinosa and L. Mott, Phys. Rev. A 68014102 (2003).

[22] P. C. Schuster and R. L. Jaffe, Ann. Phys. (N.Y.) 307132 (2003).

[23] M. V. Entin and L. I. Magarill Europhys. Lett. 68853 (2004).

[24] M. Encinosa, Phys. Rev. A 73012102 (2006).

[25] G. Cantele, D. Ninno and G. Iadonisi, Phys. Rev. B 6113730 (2000)

[26] H. Aoki, M. Koshino, D. Takeda, H. Morise and K. Kuroki, Phys. Rev. B 65 $035102(2002)$

[27] M. Koshino and H. Aoki, Phys. Rev. B 71073405 (2005)

[28] A. Marchi, S. Reggiani, M. Rudan and A. Berton, Phys. Rev. B 72035403 (2005)

[29] N. Fujita and O. Terasaki, Phys. Rev. B 72085459 (2005)

[30] R. Dandoloff and R. Balakrishman, J. Phys. A 383121 (2005)

[31] K. F. Riley, M. P. Hobson and S. J. Bence, Mathematical Methods for Physics and Engineering (Cambridge University Press) (2002)

[32] G. Parascandolo, G. Cantele, D. Ninno and G. Iadonisi, Phys. Rev. B 68245318 (2003)

[33] Y. V. Fyodorov and A. D. Mirlin Phys. Rev. Lett. 71412 (1993)

[34] H. Shima, T. Nomura and T. Nakayama Phys. Rev. B 70075116 (2004)

[35] H. Taira and H. Shima in Proceedings of the International Symposium on Topological Aspects of Critical Systems and Networks (World Scientific Pub.) in press.

[36] H. Taira and H. Shima, J. Phys. Conf. in press. 